\title{
NEW PROSPECTS FOR THE NUMERICAL CALCULATION OF MELLIN-BARNES INTEGRALS IN MINKOWSKIAN KINEMATICS*
}

\author{
IEVGEN DUBOVYK
}

\author{
II. Institut für Theoretische Physik, Universität Hamburg \\ Luruper Chaussee 149, 22761 Hamburg, Germany \\ Janusz Gluza, Tomasz Jeliński, Tord Riemann \\ Institute of Physics, University of Silesia \\ Uniwersytecka 4, 40-007 Katowice, Poland \\ JOHANN USOVITSCH \\ Institut für Physik, Humboldt-Universität zu Berlin \\ Newtonstrasse 15, 12489 Berlin, Germany
}

(Received April 7, 2017)

During the last several years, remarkable progress has been made in numerical calculations of dimensionally regulated multi-loop Feynman diagrams using Mellin-Barnes (MB) representations. The bottlenecks were non-planar diagrams and Minkowskian kinematics. The method has been proved to work in a highly non-trivial physical application (two-loop electroweak bosonic corrections to the $Z \rightarrow b \bar{b}$ decay), and cross-checked with the sector decomposition (SD) approach. In fact, both approaches have their pros and cons. For multidimensional integrals, depending on masses and scales involved, they are complementary. A powerful top-bottom approach to the numerical integration of multidimensional MB integrals is automatized in the MB-suite AMBRE/PlanarityTest/MBtools/MBnumerics/ CUBA. New key elements are: a dedicated use of the Cheng-Wu theorem for non-planar topologies and of shifts and deformations of the integration contours. An alternative bottom-up approach starting with complex 1-dimensional MB integrals, based on the exploration of steepest descent integration contours in Minkowskian kinematics, is also discussed. Shortand long-term prospects of the MB method for multi-loop applications to LHC- and LC-physics are discussed.

DOI:10.5506/APhysPolB.48.995

* Presented by J. Gluza at the Cracow Epiphany Conference "Particle Theory Meets the First Data from LHC Run 2", Kraków, Poland, January 9-12, 2017. 


\section{Introduction}

Historically, the concept of Feynman diagrams for the first time was presented by Feynman at a special by-invitation-only meeting at the Pocono Manor Inn in Pennsylvania in 1948 as an alternative to procedures of perturbative calculations in QED [1,2]. The idea was systematically treated first by Dyson in his two seminal papers [3, 4] followed by Feynman himself $^{1}[5,6]$. The integrals which stand behind the diagrams are, together with a renormalization procedure, in the core of the technical difficulties, which increase with the number of "legs" and "loops", and scales involved in calculation of contemporary QCD and electroweak processes. It is clear that steady progress in particle physics needs new ideas and crafting everchanging theoretical tools and techniques of calculations.

In the following, the MB-suite will be described in some detail. It comprises several tools for dimensionally regulated Feynman integrals in the momentum space: (i) Transform them into Feynman integrals expressed by Feynman parameters (textbook knowledge); (ii) Use the proper version of the AMBRE package [7-10] controlled for automation procedures by the PlanarityTest.m package $[11,12]$ — transform them into the Mellin-Barns integrals, valid at initial parameters which include a finite shift $\epsilon$ of dimension, $d=4-2 \epsilon$, and with original integration paths parallel to the imaginary axis; (iii) Use MB.m or MBresolve.m [13, 14] — perform an analytical continuation in $\epsilon$, approaching small $\epsilon$ and (iv) - expand the Mellin-Barnes integrals as series in small $\epsilon ;(v)$ Use barnesroutines.m from the MBtools web page [15] perform simplifications using Barnes lemmas. (vi) At this stage, the original representation of the Feynman integral in terms of several finite MB integrals has been formulated. One may now start to calculate them, either analytically or numerically, or in a mixed approach. In sufficiently complicated situations, only numerics can be applied. (vii) Use MBnumerics.m [16] to perform parametric integrations of the MB integrals along the paths defined in step (iii), thereby applying a variety of techniques: integration variable transformations, reparameterizations, contour deformations, contour shifts and whatsoever. For the parameter integrations, CUHRE of the package CUBA [17] is used. To some extent, we gave descriptions of details before $[9,18,19]$.

In this article, we focus on the purely numerical approaches to Feynman integrals developed in last few years beyond one-loop (NLO) perturbation. We show the first completed and non-trivial application in cutting-edge physical calculations using the MB-suite followed by further perspectives. In general, one is faced in numerical calculations with several technical obstacles. There are infrared singularities. We know about two methods to treat

\footnotetext{
1 That is why initially it was being called the Feynman-Dyson approach to QED.
} 
them properly without limitations. One is the MB method, the other one sector decomposition [20,21], which is also numerical. We focus here on the MB method, aiming at direct calculations in Minkowskian kinematics, which presents serious convergence problems but is crucial for production processes at high-energy accelerators such as LHC and LC. No doubt that armed with powerful fast, stable, accurate and universal software, direct numerical calculations will become possible for practical applications on "mass production", similarly as it happened at the NLO level', see FeynArts/FormCalc [35, 36], CutTools [37], Blackhat [38], Helac-1loop [39], NGluon [40], Samurai [41], Madloop [42], GoSam [43], PJFry [44], OpenLoops [45] and [46-49].

\section{Numerical concepts beyond NLO level}

Fully numerical techniques for the evaluation of two- and higher-loop integrals need the extraction of ultraviolet, infrared and collinear singularities. On top of that, they must be numerically stable and efficient. A qualitative comparison of different numerical integration techniques for Feynman parameter integration of massive multi-loop integrals can be found in [5053]. The main methods are dispersion relations, the Bernstein-Tkachov method, differential equations, the use of subtraction terms, the SD and MB methods. Here, we will discuss the last two, specifically focusing on MB. There are presently only few public programs for the numerical integration of integrals beyond the NLO level. NICODEMOS [54] is based on contour deformations. There are also complete programs dedicated specifically to the precise calculation of two-loop self-energy diagrams [55, 56]. However, the most advanced and universal programs are based on the SD or MB approaches: Sector decomposition, developed in two independent packages (present versions) Fiesta 4 [57] and SecDec 3 [21] followed by pySecDec [58]; the Mellin-Barnes method with the AMBRE project (construction of MB representations [7-10]) and PlanarityTest.m (recognition of planarity of Feynman diagrams [11, 12]) packages, followed by the MB.m [13] and MBresolve.m [14] packages for extraction of $\epsilon$-singularities in dimensional regularization of MB multiloop integrals. They offer also possibilities of numerical integrations in Euclidean kinematics, which is relatively simple as no physical branch cuts are present there. It was used intensively in the past

${ }^{2}$ To solve the integrals, analytical methods can be used, though they exhibit natural limitations when sophisticated integrals with many parameters appear. Such a situation takes place in gauge theories, like in the electroweak-QCD Standard Model. However, concerning analytical approaches to Feynman integrals, we should especially appreciate recent progress in differential equation method [22-25], which got a push in 2013 [26] followed by latest corresponding software and ideas [27-32]. Here, further progress in developing integration-by-parts (IBP) concepts is also very important [33, 34]. 
to cross-check numerically analytical results for multiloop integrals. In the next section, we will discuss new ideas which enable us to perform numerical MB integrations directly in the physical region.

\section{Numerical integrations of MB integrals in the Minkowskian region}

The Mellin-Barnes transformation of multidimensional Feynman integrals to multivariable complex contour integrations $[59,60]$ has been used in many particle physics calculations. In the first applications [61, 62] this kind of transformation has been applied directly to propagators in the loop integrals, changing "momenta ${ }^{2}$-mass" ${ }^{2}$ " terms into ratios of momenta and masses in the complex plane. Nowadays, a more efficient and systematic treatment of multiloop integrals goes by expressing Feynman integrals by the Symanzik polynomials $F$ and $U$ [63-65], for which the general MB formula is applied

$$
\begin{aligned}
\frac{1}{\left(A_{1}+\ldots+A_{n}\right)^{\lambda}}= & \frac{1}{\Gamma(\lambda)} \frac{1}{(2 \pi i)^{n-1}} \int_{c_{1}-i \infty}^{c_{1}+i \infty} \mathrm{d} z_{1} \ldots \int_{c_{2}-i \infty}^{c_{2}+i \infty} \mathrm{d} z_{2} \ldots \mathrm{d} z_{n} \prod_{=2}^{n} A_{i}^{z_{i}} \\
& \times A_{1}^{-\lambda-z_{2}-\ldots-z_{n}} \Gamma\left(\lambda+z_{2}+\ldots+z_{n}\right) \prod_{i=2}^{n} \Gamma\left(-z_{i}\right) .
\end{aligned}
$$

As we can see, $n$ additive terms lead to $n-1$ complex integrals. The $A_{i}$ terms correspond to kinematical parameters of the integral. A typical simple example is the 1-dimensional singular part of the 1-loop massive QED vertex $[9,13,66] \sim \int \mathrm{d} z(-s)^{-z} \Gamma^{3}(-z) \Gamma(1+z) \Gamma^{-1}(-2 z)$. Choosing properly the contour of integration can make the annoying oscillatory behavior of the term $(-s)^{-z}$ small and controllable (for $s>0$, so Minkowskian kinematic [9, 18]). Furthermore, the Gamma functions $\Gamma$ exhibit singularities either, and make the task of integral evaluations highly non-trivial.

The construction of MB integrals through Symanzik polynomials is automatized in the AMBRE project [7-10]. Using it with MB.m or MBresolve.m, IR and UV divergencies can be extracted and regulated multidimensional MB integrals are obtained [67]. On the webpage [15], more auxiliary packages with examples related to MB calculations can be found.

The first serious trial directed to the numerical integration of MB integrals in Minkowskian space-time was undertaken in [68]. The method developed there is based on simultaneous rotations of integration paths for all variables by the same angle in the complex plane and has been applied successfully to the calculation of two-loop diagrams with triangle fermion subloops for the $Z \rightarrow b \bar{b}$ formfactor [69]. Another interesting numerical application of 
MB integrals for phase-space integrations can be found in [70] and [71, 72]. There some parametric integrals are considered and transformations of MB integrals into Dirac delta constraints have been explored.

Now, we will present recent developments. First, we describe a topbottom approach, in which the MBnumerics.m package deals with multidimensional MB integrals; it was first described partly in [9] and applied in [19]. Another bottom-top approach is at the exploratory stage; optimal complex contours of MB integrations are worked out systematically for onedimensional MB integrals [66].

\subsection{Top-bottom approach - shifts, deformations and MBnumerics.m}

As we can see from (1), Gamma functions are there [60]. In Fig. 1, the real part of the $\Gamma[z]$ function is sketched. It is regular at positive $\operatorname{Re}[z]$ and has singularities for integer negative $\operatorname{Re}[z]$.

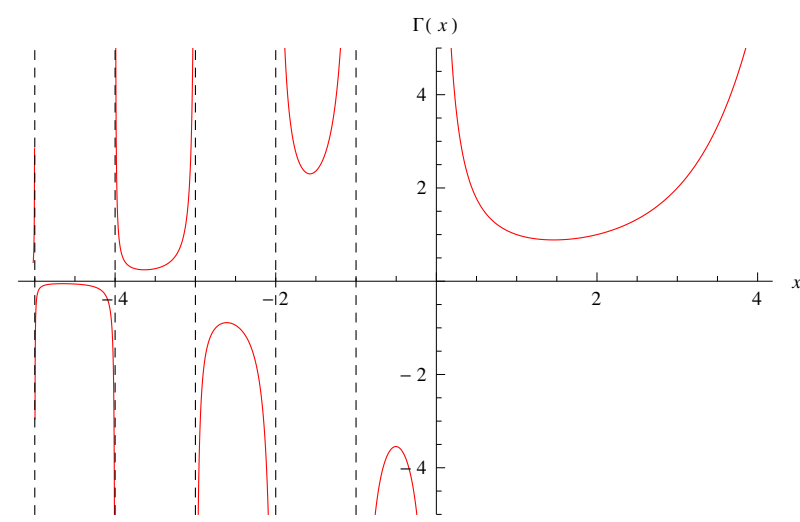

Fig. 1. Gamma function defined as $\Gamma(z)=\int_{0}^{\infty} t^{z-1} e^{-t} \mathrm{~d} t, x=\operatorname{Re}[z]$. For more details, see [73, 74].

Note also that at the negative axis between the pole positions, the integrand becomes smaller in its absolute value for the function evaluated at an argument further away from the origin. In addition, for a pole crossed by an argument shift, one has to add to the Cauchy integral (1) the corresponding residue which, by itself, is also an integral, but will have a dimension less than the original one. Repeating the procedure for several integration variables, the original MB integral gets replaced by several lower-dimensional integrals, and the original one with a shifted integration path. In the end, the (module of the) resulting contribution of the original integral after shifts can be made smaller than the desired accuracy of the calculation. In effect, the procedure constructs a sum over a finite number of residues with a controlled remainder. This procedure of shifts is implemented in MBnumerics.m 
[16]. Some other important features of the procedure such as contour deformations and mappings of parameter integrals into finite intervals have been discussed in $[9,18]$. Figure 2 sketches roughly the idea.

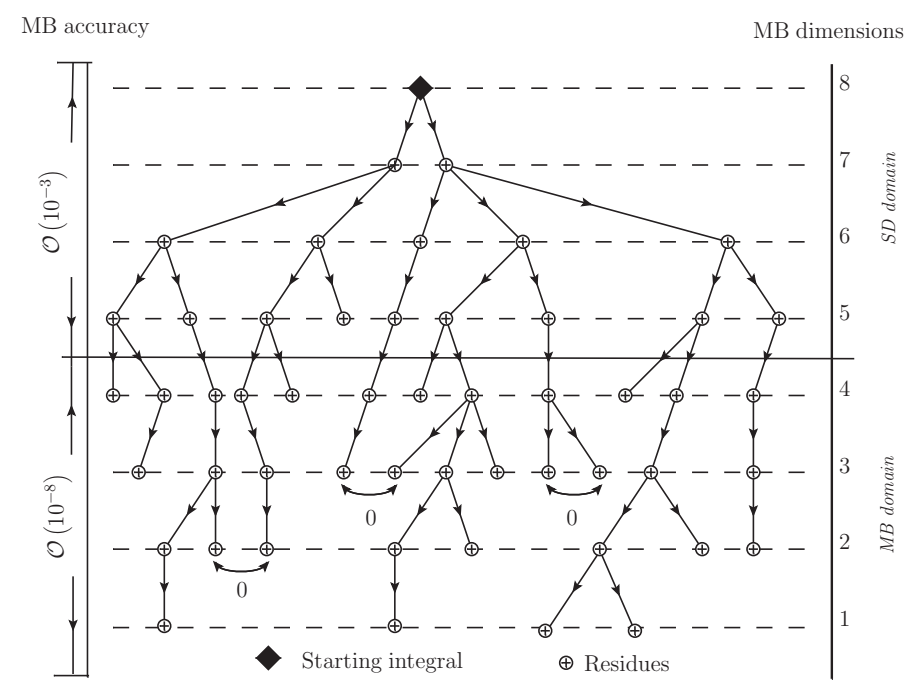

Fig. 2. A possible scenario for the calculation of some 8-dimensional MB integral. Lower dimensional MB integrals result from shifting complex variables of the integral by integers, as explained in the text. In [19], all integrals of dimension less than 5 were calculated this way with MBnumerics.m [16] and high accuracy, the remaining integrals were treated with the same accuracy by the SD method. However, as a basic cross-check, less digits could be obtained for all integrals using both methods.

In the project [19], we derived Mellin-Barnes representations for all integrals, which had up to eight dimensions. For a cross-check, each integral was computed with MB and SD techniques. There are only few classes of diagrams for which eight digits could not be achieved with both methods, an example is given in Fig. 3; for further discussion, see [9, 18]. These diagrams have high order divergences and an application of the sector decomposition approach leads to numerical problems related to both accuracy and time consumption. In contrast to this, the corresponding MB integrals can be computed with reasonable computer time resources.

Typically, for integrals which involve many masses, SD fits better while the MB method works out perfectly for more "massless" diagrams. Thus, the MB-suite and the sector decomposition techniques are, to a large extent, complementary [8] and both numerical methods can be successfully explored together in cutting-edge physical problems. 

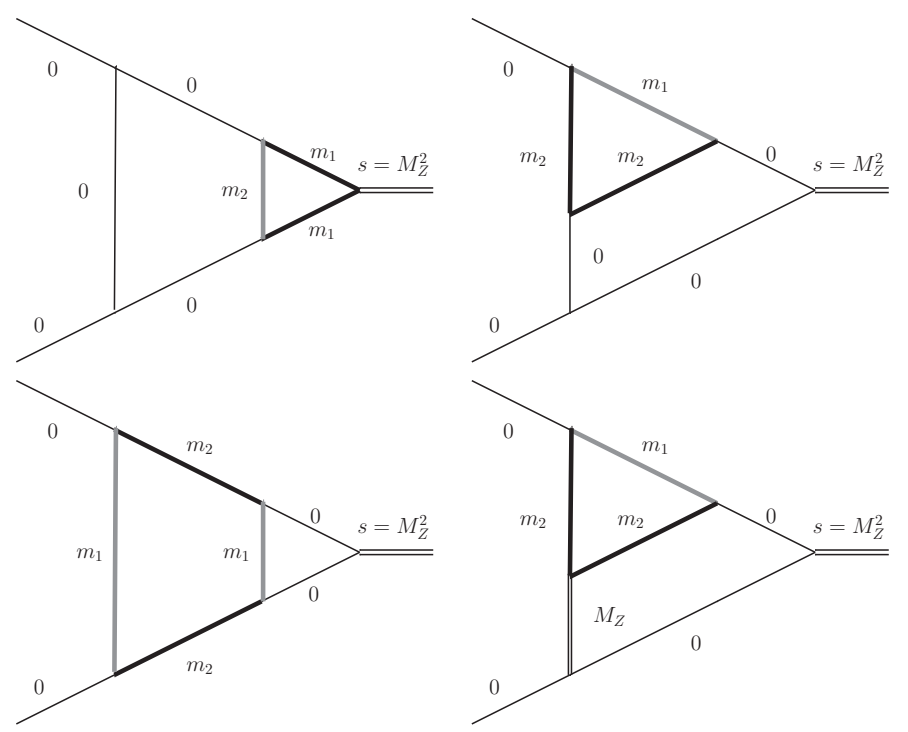

Fig. 3. Examples of diagrams appearing in the calculation of $\sin ^{2} \theta_{\text {eff }}^{b}$ [19] for which MB and SD methods have been applied. The MB representations have up to 4-dimensional integrals, to be taken at the $Z$ boson mass shell, $s=M_{Z}^{2}$. A numerical accuracy at the level of $\mathcal{O}\left(10^{-8}\right)$ was achieved for them only with the MB approach. For a general $s$-dependence, the situation is the same.

In [19] for MB and planar diagrams, the newest version AMBRE v2.1 [10] is used, for non-planar diagrams it is AMBRE v3.1 [10]. The planarity of diagrams is controlled automatically with the PlanarityTest.m package $[11,12]$. Numerical results have been obtained using MBnumerics.m [16]. As it is demonstrated in Fig. 2, the shifts accumulate at each new iteration many residues, until the desired accuracy is reached. It is worth noting that the integration error of MBnumerics.m is mostly dominated by the collection of residues which have fast convergence. For higher-dimensional integrals, MBnumerics.m collects more residues. The resulting error from all residues is determined by Pythagorean addition. In Fig. 2, the two-sided arrows with label "0" denote pairs of residues which are identified to finally exactly cancel. To identify such pairs to a high accuracy, MBnumerics.m performs the integration of the corresponding candidates at a different kinematical point where a high numerical accuracy is reached. If then the integrals agree up to a sign, MBnumerics.m sums them up to zero. This is only one example of many numerical problems which have been solved in the MBnumerics.m algorithm in order to get highly accurate numerical results in the Minkowskian region. The package is yet under development, and our present estimation is that in the near future, even 12-dimensional MB integrals can be touched - e.g. pentaboxes. 


\subsection{Bottom-top MBDE approach - optimal steepest descent contours}

In a nutshell, this is a stationary phase method leading to optimal steepest descent integration contours. They can be found using Lefschetz thimbles (exact contours) or their Padé approximation [66].

Lefschetz thimbles (LT) are a fascinating subject, crossing many issues such as behaviour of LT in presence of poles, singularities and branch cuts, behaviour in the complex infinity, Stokes phenomenon, relation to relative homology of a punctured Riemann sphere, etc. It can be applied e.g. to the analytical continuation of 3d Chern-Simons theory, QCD with chemical potential, resurgence theory, counting master integrals or the repulsive Hubbard model. Still, applying this method to the numerical evaluation of MB integrals is at the exploratory stage, and an effective and general determination of multivariate MB contours must be worked out yet in more detail.

In this section, we present the main idea as an alternative approach to the numerical computation of MB integrals, starting from the bottom, the lowest one-dimensional MB integrals, in both Euclidean $(s<0)$ and Minkowski $(s>0)$ regions. These cases have been explored in fine details in [66].

Let us write a general MB integrand $F(z)$, transformed into exponential form $^{3}$

$$
I(s)=\frac{1}{2 \pi i} \int_{\mathcal{C}_{0}} \mathrm{~d} z F(z)=\frac{1}{2 \pi i} \int_{c_{0}-i \infty}^{c_{0}+i \infty} \mathrm{d} z e^{-f(z)} .
$$

$\mathcal{C}_{0}$ is a contour defined by $\operatorname{Re} z=c_{0}$, while $f(z)=-\ln F(z)$.

One of the possible ways to get rid of numerical problems with the MB integrand $F(z)$ which is of highly-oscillatory behaviour [9] is to integrate (2) over a new contour $\mathcal{C}=\mathcal{J}_{1}+\mathcal{J}_{2}+\mathcal{A}$. A typical example is sketched in Fig. 4 where $\mathcal{C}$ is a sum of three contours $\mathcal{J}_{1}, \mathcal{J}_{2}$ and $\mathcal{A}$ along which the behaviour of $f$ is under control.

Taking $f=\operatorname{Re} f+i \operatorname{Im} f$, we deform the original integration contour $\mathcal{C}_{0}$ to a Lefschetz thimble $\mathcal{J}\left(z_{*}\right)$

$$
\int_{\mathcal{C}_{0}} \mathrm{~d} z e^{-f}=\overbrace{e^{-\left.i \operatorname{Im} f\right|_{\mathcal{J}\left(z_{*}\right)}}}^{\text {Overall factor }} \overbrace{\int_{\mathcal{J}\left(z_{*}\right)} \mathrm{d} z e^{-\operatorname{Re} f}}^{\text {Damping factor }}+\overbrace{2 \pi i \sum_{\mathcal{C}_{0} \rightarrow \mathcal{J}\left(z_{*}\right)} \operatorname{Res} e^{-f}}^{\text {Remnants }} .
$$

The analytical formula describing $\mathcal{J}_{k}$ can be found only in the simplest cases by explicit solving the equation $\operatorname{Im} f=$ const. Instead, we use the fact that the function $\operatorname{Re} f$ defines a Morse flow [75, 76]. Such a flow is

\footnotetext{
${ }^{3}$ For brevity, we suppress the dependence on $s$ and shall use $F(z)$ instead of $F(s, z)$.
} 
realized by a parametrization $t \mapsto z(t)$ of $\mathcal{J}_{k}\left(z_{*}\right)$ in a form of Lefschetz thimbles [77-82]. The Lefschetz thimble $\mathcal{J}\left(z_{*}\right)$ is defined as a union of curves $t \rightarrow z(t)=\left(z_{1}(t), \ldots, z_{i}(t), \ldots, z_{n}(t)\right) \in \mathbb{C}^{n}$ which satisfy the following differential equation [78, 81]:

$$
\frac{\mathrm{d} z_{i}(t)}{\mathrm{d} t}=-\left(\frac{\partial f(z)}{\partial z^{i}}\right)^{*}, \quad z(+\infty)=z_{*} .
$$

Here, $z_{*}$ is a saddle point of a meromorphic function $f$. The crucial observation is that for $\mathcal{J}\left(z_{*}\right)$ we can take $\operatorname{Im} f=$ const, leading to the overall factor in (3). Note that $\operatorname{Im} f$ generates a Hamiltonian flow on $\mathbb{R}^{2 n}$, e.g. for $n=1$,

$$
\frac{\mathrm{d} x(t)}{\mathrm{d} t}=\frac{\partial \operatorname{Im} f}{\partial y}, \quad \frac{\mathrm{d} y(t)}{\mathrm{d} t}=-\frac{\partial \operatorname{Im} f}{\partial x} .
$$

The $\operatorname{Re} f$ is monotonically decreasing when $t \rightarrow+\infty$ and goes to $+\infty$ when $t \rightarrow-\infty$, leading to the damping factor in (3).

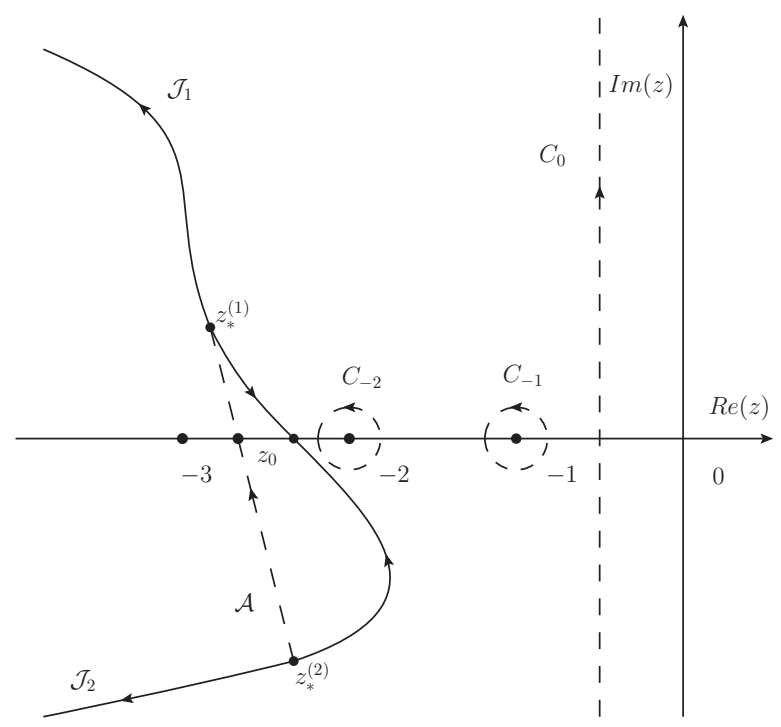

Fig. 4. A deformation of the integration contour $\mathcal{C}_{0}$ defined by $\operatorname{Re} z=c_{0}$ to a contour $\mathcal{C}=\mathcal{J}_{1}+\mathcal{J}_{2}+\mathcal{A}$. $\mathcal{J}_{1,2}$ are two Lefschetz thimbles which start at saddle points $z_{*}^{(1,2)}$ and go towards infinity. The compact contour $\mathcal{A}$ (interval) connects the two saddle points $z_{*}^{(1)}$ and $z_{*}^{(2)}$. When there is an obstruction in deriving the parametrization of $\mathcal{J}_{1,2}$ around some point, e.g. $z_{0}$, one can bypass that region using the contour $\mathcal{A}$. Note that here a deformation $\mathcal{C}_{0} \rightarrow \mathcal{C}$ requires taking into account integrals over two "small" contours, $\mathcal{C}_{-2}$ and $\mathcal{C}_{-1}$ around poles at $z=-2$ and $z=-1$ which contribute to $\sum \operatorname{Res} F$ in (3). 
Remnants in (3) are, according to Cauchy's theorem, residues over poles when the integration contour is deformed from $\mathcal{C}_{0}$ to $\mathcal{C}$ and encircles extra poles of $F=e^{-f}$, as shown in Fig. 4. In this way, (4) gives a possibility to solve for $z(t)$ such that integral (3) is under control. As $\mathcal{J}_{1,2}$, we choose such stationary phase contours which start at saddle points $z_{*}^{(1,2)}$ and go towards infinity without hitting other poles. Both contours are chosen such that $\operatorname{Im} f$ is constant along them and function $\operatorname{Re} f$ is strictly increasing when one moves away from $z_{*}^{(1,2)}$. Varieties defined in such a way are called steepest descent contours [83, 84]. Usage of $\mathcal{J}_{1,2}$ allows to control the behaviour of $f(z)$, when $z \rightarrow \infty$. Because $\operatorname{Re} f$ is strictly increasing, the integrand $e^{-f}$ decreases rapidly at the ends of $\mathcal{J}_{1,2}$. That transforms integral (2) into a form which is more suitable for a numerical treatment.

With respect to various methods known in the literature [9, 13, 52, 68] which shift/rotate contours or use approximate forms thereof, the MB method which relies on the differential equation (4), in short the MBDE method, relies on deriving the numerical parametrization $z(t)$ of $\mathcal{J}_{k}$ as a solution of the differential equation (4) and then, again numerically, integrating the function $e^{-\operatorname{Re} f}$ along the contour $\mathcal{C}$ composed of Lefschetz thimbles $\mathcal{J}_{k}$ (and the compact contour $\mathcal{A}$ if necessary). The purely numerical approach MBDE is complementary to the Padé approximation presented in [66].

Let us shortly discuss numerical features of the MBDE method and display results of some performance tests. We stress that the tests are preliminary, implemented directly in Mathematica, in graphical mode, on an i7 2.9 $\mathrm{GHz}$ CPU. Both kinematical regions $s<0$ and $s>0$ are treated in the same way in MBDE, although $s>0$ seems to be more CPU time consuming. For a final accuracy of the order of $10^{-6}$, the method is as fast as MB [13] and MBnumerics $[9,16]$, while for an accuracy of $10^{-11}$ and higher, MBDE turns out to be more than 10 times slower than the other two packages; see Table I. To get a precision of the order of $10^{-16}$, some kind of optimization

TABLE I

Performance tests of MBDE for the integrand $F_{1}(z)=(-s)^{-z} \Gamma^{3}(-z) \Gamma(z+1) /$ $\Gamma(-2 z)$. The relative error $\delta_{\mathrm{an}}$ is defined as $\delta_{\mathrm{an}}=\left|\left(I_{\mathrm{an}}-I_{\mathrm{MBDE}}\right) / I_{\mathrm{an}}\right| . \quad I_{\mathrm{an}}$ is the analytical value of the integral $I_{1}(s), I_{\mathrm{MBDE}}$ is the numerical value of this integral evaluated with the MBDE method. Finally, $T_{\mathrm{MBDE}, \mathrm{MB}, \mathrm{MBnum}}$ display runtimes (in seconds) needed to numerically evaluate an integral using MBDE, MB.m and MBnumerics.m (with default settings), respectively.

\begin{tabular}{c|c|c|c|c|c}
\hline \hline$s$ & $I_{\mathrm{MBDE}}$ & $-\log _{10} \delta_{\mathrm{an}}$ & $T_{\mathrm{MBDE}}[\mathrm{s}]$ & $T_{\mathrm{MB}}[\mathrm{s}]$ & $T_{\mathrm{MBnum}}[\mathrm{s}]$ \\
\hline$-1 / 20$ & $4.96 \times 10^{-2}$ & $6 / 9 / 11$ & $1.31 / 2.48 / 16.94$ & 1.43 & 1.16 \\
$1+i 0^{ \pm}$ & -1.21 & $6 / 11$ & $15.05 / 53.19$ & - & 1.28 \\
5 & $4.30+14.05 i$ & 10 & 13.5 & - & 1.57
\end{tabular}


of the method is needed. Presumably, it can be made much faster by implementing in e.g. Fortran or $\mathrm{C} / \mathrm{C}++$ or by applying a dedicated method of solving differential equations. Parallelization or dividing integration regions into smaller parts to achieve larger precision are also possible options.

\section{Summary and outlook}

In the last few years, substantial progress was obtained in the direct calculation of multiloop integrals (Feynman diagrams) in the physical, Minkowski regime using both the SD and MB methods. The methods are complementary in several respects. In the MB case, the most advanced is the topbottom approach implemented in the MB-suit, where multidimensional MB integrals can be solved in physical kinematics with high accuracy for MB integrals of dimension eight and below. Potential applications of the discussed numerical methods are complete 2-loop electroweak pseudoobservables needed for future linear colliders - multi-massive 2-loop vertices, and also non-resonant two-loop box diagrams, and complete cross sections, including LHC problems [68].

Using numerical methods, we are approaching automation in calculation of Feynman integrals beyond the NLO level directly in physical kinematics. The perspectives are robust, concerning both high- and low-energy physics. Seemingly, the "NNLO revolution" is emerging, quite similar to the NLO revolution of the last decade.

I.D. is supported by a research grant of Deutscher Akademischer Austauschdienst (DAAD) and by Deutsches Elektronensychrotron DESY; J.G. and T.J. are supported by the Polish National Science Centre (NCN) under the grant agreement No. DEC-2013/11/B/ST2/04023; T.R. is supported in part by an Alexander von Humboldt Polish Honorary Research Fellowship. The work of J.U. is supported by Graduiertenkolleg 1504 "Masse, Spektrum, Symmetrie" of Deutsche Forschungsgemeinschaft (DFG).

\section{REFERENCES}

[1] S.S. Schweber, QED and the Men Who Made It: Dyson, Feynman, Schwinger, and Tomonaga, Princeton University Press, Princeton 1994, p. 732 .

[2] D. Kaiser, Drawing Theories Apart: the Dispersion of Feynman Diagrams in Postwar Physics, Chicago University Press, Chicago, IL, 2005.

[3] F.J. Dyson, Phys. Rev. 75, 486 (1949).

[4] F.J. Dyson, Phys. Rev. 75, 1736 (1949).

[5] R.P. Feynman, Phys. Rev. 76, 749 (1949). 
[6] R.P. Feynman, Phys. Rev. 76, 769 (1949).

[7] J. Gluza, K. Kajda, T. Riemann, Comput. Phys. Commun. 177, 879 (2007) [arXiv:0704.2423 [hep-ph]].

[8] J. Gluza, K. Kajda, T. Riemann, V. Yundin, Eur. Phys. J. C 71, 1516 (2011) [arXiv:1010.1667 [hep-ph]].

[9] I. Dubovyk, J. Gluza, T. Riemann, J. Usovitsch, PoS LL2016, 034 (2016) [arXiv: 1607.07538 [hep-ph]].

[10] University of Silesia in Katowice webpage: http://prac.us.edu.pl/〜gluza/ambre

[11] K. Bielas, I. Dubovyk, PlanarityTest.m, a Mathematica package for testing the planarity of Feynman diagrams, http://us.edu.pl/〜gluza/ambre/planarity/

[12] K. Bielas, I. Dubovyk, J. Gluza, T. Riemann, Acta Phys. Pol. B 44, 2249 (2013) [arXiv:1312.5603 [hep-ph]].

[13] M. Czakon, Comput. Phys. Commun. 175, 559 (2006) [arXiv:hep-ph/0511200].

[14] A. Smirnov, V. Smirnov, Eur. Phys. J. C 62, 445 (2009) [arXiv:0901.0386 [hep-ph]].

[15] M. Czakon (MB, MBasymptotics), D. Kosower (barnesroutines), A. Smirnov, V. Smirnov (MBresolve), K. Bielas, I. Dubovyk, J. Gluza, K. Kajda, T. Riemann (AMBRE, PlanarityTest), MBtools webpage, https://mbtools.hepforge.org/

[16] I. Dubovyk, T. Riemann, J. Usovitsch, MBnumerics.m 1.0, a Mathematica package for numerical evaluation of Mellin-Barnes integrals in Minkowskian regions, in preparation.

[17] T. Hahn, Comput. Phys. Commun. 168, 78 (2005) [arXiv:hep-ph/0404043].

[18] I. Dubovyk et al., PoS LL2016, 075 (2016) [arXiv:1610.07059 [hep-ph]].

[19] I. Dubovyk et al., Phys. Lett. B 762, 184 (2016) [arXiv:1607.08375 [hep-ph]].

[20] A.V. Smirnov, Comput. Phys. Commun. 185, 2090 (2014) [arXiv:1312.3186 [hep-ph]].

[21] S. Borowka et al., Comput. Phys. Commun. 196, 470 (2015) [arXiv: 1502.06595 [hep-ph]].

[22] A.V. Kotikov, Phys. Lett. B 254, 158 (1991).

[23] A.V. Kotikov, Phys. Lett. B 259, 314 (1991).

[24] A.V. Kotikov, Phys. Lett. B 267, 123 (1991).

[25] E. Remiddi, Nuovo Cim. A 110, 1435 (1997) [arXiv:hep-th/9711188].

[26] J.M. Henn, Phys. Rev. Lett. 110, 251601 (2013) [arXiv:1304.1806 [hep-th]].

[27] J. Ablinger et al., Comput. Phys. Commun. 202, 33 (2016) [arXiv: 1509.08324 [hep-ph]].

[28] M. Prausa, arXiv:1701.00725 [hep-ph]. 
[29] O. Gituliar, V. Magerya, arXiv:1701.04269 [hep-ph].

[30] A. von Manteuffel, L. Tancredi, arXiv:1701.05905 [hep-ph].

[31] C. Meyer, arXiv:1705.06252 [hep-ph].

[32] L. Adams, E. Chaubey, S. Weinzierl, Phys. Rev. Lett. 118, 141602 (2017) [arXiv:1702.04279 [hep-ph]].

[33] P. Maierhoefer, J. Usovitsch, P. Uwer, arXiv:1705.05610 [hep-ph].

[34] A. Georgoudis, K.J. Larsen, Y. Zhang, arXiv:1612.04252 [hep-th].

[35] T. Hahn, Comput. Phys. Commun. 140, 418 (2001) [arXiv: hep-ph/0012260].

[36] B. Chokoufe Nejad, T. Hahn, J.N. Lang, E. Mirabella, J. Phys.: Conf. Ser. 523, 012050 (2014) [arXiv:1310.0274 [hep-ph]].

[37] G. Ossola, C.G. Papadopoulos, R. Pittau, J. High Energy Phys. 0803, 042 (2008) [arXiv:0711.3596 [hep-ph]].

[38] C.F. Berger et al., Phys. Rev. D78, 036003 (2008) [arXiv:0803.4180 [hep-ph]].

[39] A. van Hameren, C.G. Papadopoulos, R. Pittau, J. High Energy Phys. 0909, 106 (2009) [arXiv:0903.4665 [hep-ph]].

[40] S. Badger, B. Biedermann, P. Uwer, Comput. Phys. Commun. 182, 1674 (2011) [arXiv: 1011.2900 [hep-ph]].

[41] P. Mastrolia, G. Ossola, T. Reiter, F. Tramontano, J. High Energy Phys. 1008, 080 (2010) [arXiv:1006.0710 [hep-ph]].

[42] V. Hirschi et al., J. High Energy Phys. 1105, 044 (2011) [arXiv:1103.0621 [hep-ph]].

[43] G. Cullen et al., Eur. Phys. J. C 72, 1889 (2012) [arXiv:1111.2034 [hep-ph]].

[44] J. Fleischer, T. Riemann, V. Yundin, PoS LL2012, 020 (2012) [arXiv:1210.4095 [hep-ph]].

[45] F. Cascioli, P. Maierhofer, S. Pozzorini, arXiv:1111.5206 [hep-ph].

[46] G. van Oldenborgh, Comput. Phys. Commun. 66, 1 (1991).

[47] A. van Hameren, Comput. Phys. Commun. 182, 2427 (2011) [arXiv:1007.4716 [hep-ph]].

[48] R.K. Ellis, G. Zanderighi, J. High Energy Phys. 0802, 002 (2008) [arXiv:0712.1851 [hep-ph]].

[49] A. Denner, S. Dittmaier, L. Hofer, Comput. Phys. Commun. 212, 220 (2017) [arXiv:1604.06792 [hep-ph]].

[50] V. Smirnov, Applied Asymptotic Expansions in Momenta and Masses, Springer Verlag, Berlin 2002.

[51] M. Steinhauser, Phys. Rep. 364, 247 (2002) [arXiv:hep-ph/0201075].

[52] C. Anastasiou, A. Daleo, J. High Energy Phys. 0610, 031 (2006) [arXiv:hep-ph/0511176]. 
[53] A. Freitas, Prog. Part. Nucl. Phys. 90, 201 (2016) [arXiv:1604.00406 [hep-ph]].

[54] A. Freitas, J. High Energy Phys. 1207, 132 (2012) [Erratum ibid. 1209, 129 (2012)] [arXiv:1205.3515 [hep-ph]].

[55] S.P. Martin, D.G. Robertson, Comput. Phys. Commun. 174, 133 (2006) [arXiv:hep-ph/0501132].

[56] M. Caffo, H. Czyż, M. Gunia, E. Remiddi, Comput. Phys. Commun. 180, 427 (2009) [arXiv:0807.1959 [hep-ph]].

[57] A.V. Smirnov, Comput. Phys. Commun. 204, 189 (2016) [arXiv: 1511.03614 [hep-ph]].

[58] S. Borowka et al., [arXiv:1703.09692 [hep-ph]].

[59] R.H. Mellin, Om Dedinita Integraler, Acta Soc. Sci. Fenn. 20, 1 (1895).

[60] E.W. Barnes, The Theory of the Gamma Function, Messenger Math. 29, 64 (1900).

[61] M.C. Bergere, Y.-M.P. Lam, Commun. Math. Phys. 39, 1 (1974).

[62] N.I. Ussyukina, Teor. Mat. Fiz. 22, 300 (1975).

[63] V.A. Smirnov, Phys. Lett. B 460, 397 (1999) [arXiv:hep-ph/9905323].

[64] J. Tausk, Phys. Lett. B 469, 225 (1999) [arXiv:hep-ph/9909506].

[65] V. Smirnov, Evaluating Feynman Integrals, Springer Verlag, Berlin 2004.

[66] J. Gluza, T. Jeliński, D.A. Kosower, Phys. Rev. D 95, 076016 (2017) [arXiv:1609.09111 [hep-ph]].

[67] V. Smirnov, Springer Tracts Mod. Phys. 211, 1 (2004).

[68] A. Freitas, Y.-C. Huang, J. High Energy Phys. 1004, 074 (2010) [arXiv:1001.3243 [hep-ph]].

[69] A. Freitas, Y.-C. Huang, J. High Energy Phys. 1208, 050 (2012) [Errata ibid. 1305, 074 (2013), 1310, 044 (2013)] [arXiv:1205.0299 [hep-ph]].

[70] G. Somogyi, J. Math. Phys. 52, 083501 (2011) [arXiv:1101.3557 [hep-ph]].

[71] C. Anastasiou, S. Beerli, A. Daleo, J. High Energy Phys. 0705, 071 (2007) [arXiv:hep-ph/0703282].

[72] C. Anastasiou, C. Duhr, F. Dulat, B. Mistlberger, J. High Energy Phys. 1307, 003 (2013) [arXiv:1302.4379 [hep-ph]].

[73] E.W. Barnes, Proc. Lond. Math. Soc. (2) 6, 141 (1908).

[74] E. Whittaker, G. Watson, A Course of Modern Analysis, Cambridge University Press, 1965.

[75] L. Nicolaescu, An Invitation to Morse Theory, Universitext, Springer, New York 2011.

[76] V. Arnold, A. Varchenko, S. Gusein-Zade, Singularities of Differentiable Maps: Volume II Monodromy and Asymptotic Integrals, Monographs in Mathematics, Birkhäuser, Boston 2012. 
[77] F. Pham, Vanishing Homologies and the $n$ Variable Saddlepoint Method, Proc. Symp. Pure Math., Vol. 40(2), AMS (1983), pp. 319-333.

[78] E. Witten, AMS/IP Stud. Adv. Math. 50, 347 (2011) [arXiv:1001.2933 [hep-th]].

[79] E. Witten, arXiv:1009.6032 [hep-th].

[80] D. Harlow, J. Maltz, E. Witten, J. High Energy Phys. 1112, 071 (2011) [arXiv:1108.4417 [hep-th]].

[81] T. Kanazawa, Y. Tanizaki, J. High Energy Phys. 1503, 044 (2015) [arXiv:1412.2802 [hep-th]].

[82] Y. Tanizaki, T. Koike, Ann. Phys. 351, 250 (2014) [arXiv:1406.2386 [math-ph]].

[83] C. Bender, S. Orszag, Advanced Mathematical Methods for Scientists and Engineers I. Asymptotic Methods and Perturbation Theory, Springer, 1999.

[84] R. Wong, Asymptotic Approximation of Integrals, Series: Classics in Applied Mathematics, Society for Industrial and Applied Mathematics, 2001. 